\title{
Biotechnologies et conservation des ressources phytogénétiques
}

\section{Florent Engelman}

IRD

911, avenue agropolis BP 64501

34394 Montpellier

France

<engelman@ird.fr>

\begin{abstract}
Résumé
Les techniques de culture in vitro ont de nombreuses applications pour la collecte, la propagation et la conservation de la biodiversité végétale. Les techniques de collecte in vitro permettent l'introduction in vitro sur le terrain d'explants d'espèces à semences récalcitrantes et propagées végétativement. Les techniques de culture in vitro permettent la production et la multiplication à grande échelle de matériel sain. L'utilisation des techniques de culture in vitro est d'un grand intérêt pour conserver : 1) les ressources génétiques d'espèces à semences récalcitrantes et propagées végétativement ; 2) les produits de la biotechnologie (génotypes élite, lignées cellulaires productrices de métabolites, matériel génétiquement modifié) ; 3) les espèces rares et menacées. La conservation à moyen terme est réalisée en réduisant la croissance du matériel végétal, ce qui permet d'espacer les repiquages. Des protocoles de stockage en croissance ralentie sont employés pour de nombreuses espèces tropicales et tempérées. Pour la conservation à long terme, la cryoconservation (azote liquide, $-196^{\circ} \mathrm{C}$ ) permet de stocker le matériel végétal sans modifications ni altérations pendant des durées prolongées, à l'abri des contaminations et avec un entretien réduit. La cryoconservation est bien développée avec les espèces à multiplication végétative. Les recherches sont nettement moins avancées avec les espèces à semences récalcitrantes, du fait de certaines de leurs caractéristiques, telles leur sensibilité élevée à la dessiccation, leur complexité structurale et leur hétérogénéité en termes de stades de développement et de teneur en eau à maturité. Cependant, diverses approches existent afin de mettre au point des techniques de cryoconservation pour un nombre plus élevé d'espèces à semences récalcitrantes. L'utilisation en routine de la cryoconservation est encore limitée. Cependant le nombre d'exemples pour lesquels elle est utilisée à grande échelle est en augmentation constante.
\end{abstract}

Mots clés : biotechnologie végétale ; conservation des ressources ; cryoconservation ; culture in vitro ; ressource génétique végétale.

Thèmes : productions végétales ; méthodes et outils ; ressources génétiques et environnement.

\section{Abstract \\ Biotechnologies and conservation of phytogenetic resources}

In vitro techniques have numerous applications in collecting, propagating and conserving plant biodiversity. In vitro collecting techniques make it possible to introduce in vitro explants under field conditions from recalcitrant seeds and vegetatively propagated species. In vitro culture techniques make production and large scale propagation of disease-free material possible. Utilising in vitro culture techniques is of high interest for conserving: 1) genetic resources of recalcitrant seeds and vegetatively propagated species; biotechnology products (elite genotypes, metabolite-producing cell lines, transformed material) and 3) rare and endangered plant species. Medium term conservation is performed by reducing growth of the plant material, thereby increasing intervals between subcultures. Slow growth storage protocols are employed for numerous temperate and tropical species. For long term storage, cryopreservation (liquid nitrogen $-196{ }^{\circ} \mathrm{C}$ ) makes it possible to store plant material without modification or alteration for extended periods, sheltered from contamination and with limited maintenance. Cryopreservation is well advanced for vegetatively propagated plant species. Research is less advanced for recalcitrant species due to certain characteristics including their sensitivity to desiccation, their structural complexity and heterogeneity in terms of developmental stages and their water content at maturity. However, various technical approaches exist 
in order to establish cryopreservation techniques for a larger number of recalcitrant species. Routine utilisation of cryopreservation is still limited. However, the number of examples for which it is used on a large scale is increasing steadily.

Key words: cryopreservation; in vitro culture; plant biotechnology; plant genetic resources; resource conservation.

Subjects: natural resources and environment; tools and methods; vegetal productions.

$\grave{A}$ l'aube du xxI ${ }^{\mathrm{e}}$ siècle, l'agriculture est confrontée à de nouveaux défis, afin d'assurer une production et une autosuffisance alimentaire durables, et de contribuer à l'éradication de la pauvreté et à l'équilibre écologique de la planète. Les agriculteurs sont en première ligne, en particulier au travers des variétés de plantes et des semences qu'ils conservent. Cependant, la communauté scientifique impliquée dans la conservation de la biodiversité et des ressources génétiques végétales a également un rôle crucial à jouer. Ses travaux doivent permettre une adaptation rapide des espèces végétales cultivées aux changements à venir, qui comprennent l'érosion de la biodiversité, la dégradation de l'environnement, les changements climatiques et les risques associés ainsi que la diversification de l'utilisation des produits agricoles. Cette adaptation reposera sur l'identification et l'utilisation de caractères spécifiques qui sont présents dans les collections de ressources génétiques végétales qui existent dans le monde entier.

Parmi les plantes alimentaires majeures, de nombreuses espèces végétales produisent des semences qui présentent une phase de déshydratation importante en fin de maturation. Elles sont donc tolérantes à une déshydratation intense et peuvent être conservées à basse température à l'état déshydraté. Les semences de ces espèces sont nommées orthodoxes (Roberts, 1973). Le stockage des semences orthodoxes est la méthode la plus largement utilisée de conservation ex situ des ressources phytogénétiques, puisque $90 \%$ des 6,1 millions d'accessions stockées dans les banques de gènes sont conservées sous la forme de semences. Par opposition aux semences orthodoxes, un nombre considérable d'espèces, principalement d'origine tropicale ou subtropicale - telles que le cocotier, le cacaoyer ou de très nombreux arbres fruitiers ou forestiers - produisent des semences qui ont une phase de déshydratation très légère en fin de maturation et qui sont donc disséminées à des teneurs en eau relativement élevées. De telles semences ne peuvent résister à la déshydratation et sont souvent sensibles au froid. Elles ne peuvent donc pas être conservées dans les conditions de stockage traditionnelles des semences, c'est-à-dire à teneur en eau réduite et à basse température. Ces semences sont appelées récalcitrantes et doivent être conservées dans des conditions d'humidité et de température relativement élevées pour maintenir leur viabilité (Roberts, 1973). Même lorsqu'elles sont stockées dans des conditions optimales, leur viabilité est limitée à quelques semaines ou mois. Il existe d'autres espèces dont la conservation sous forme de semences pose des problèmes. Tout d'abord, ce sont les espèces qui ne produisent pas de semences et qui sont par conséquent propagées de manière végétative, telles que le bananier et le plantain (Musa spp.). Par ailleurs, des espèces comme la pomme de terre (Solanum tuberosum), d'autres racines et tubercules comme l'igname (Dioscorea spp.), le manioc (Manibot esculenta) et la patate douce (Ipomoea batatas), ainsi que la canne à sucre (Saccharum spp.), ont soit des génotypes stériles, soit des génotypes qui produisent des semences orthodoxes. Cependant, ces semences sont hautement hétérozygotes et elles sont donc d'une utilité limitée pour la conservation de génotypes particuliers. Ces plantes sont généralement propagées de manière végétative pour maintenir les génotypes sous la forme de clones.

La méthode de conservation ex situ traditionnelle de ces espèces dont la conservation pose des problèmes est la conservation sous forme de collections en champ. Cependant, cette méthode, si elle offre des avantages certains, présente des inconvénients qui limitent son efficacité et menacent sa sécurité (Engelmann, 1997). Les ressources génétiques de ces espèces sont exposées aux ravageurs et aux maladies, aux calamités naturelles comme la sécheresse ou les ouragans, aux erreurs humaines et au vandalisme. De plus, elles ne sont pas sous une forme qui facilite les échan- ges de matériel génétique, à cause des risques élevés de transfert de maladies lors de l'échange de matériel végétatif. Les collections en champ sont coûteuses à maintenir et, par conséquent, elles sont à la merci de décisions économiques qui peuvent limiter le niveau de réplication des accessions, la qualité de leur entretien et même leur survie en cas de difficultés économiques. Même dans les meilleures conditions, les collections en champ nécessitent des intrants considérables sous forme de terrain, main-d'œuvre, gestion, et matériel. De plus, leur capacité à conserver une quantité importante de diversité est limitée.

Au vu de ces problèmes, il n'est pas surprenant que des efforts aient été faits pour améliorer la qualité et la sécurité de la conservation offerte par les collections en champ et pour comprendre et régler les problèmes causés par la récalcitrance des semences, afin de rendre le stockage des semences plus largement disponible. Cependant, il est clair que des approches alternatives sont nécessaires pour la conservation des ressources génétiques des matériels qui posent des problèmes : depuis les années 1970, l'attention s'est tournée vers les possibilités offertes par les biotechnologies et, de manière plus spécifique, par la culture in vitro et la cryoconservation.

\section{Techniques biotechnologiques développées pour la conservation de la biodiversité végétale}

$\mathrm{Au}$ cours des 30 dernières années, les techniques de culture in vitro se sont lar- 
gement développées et elles ont été appliquées à plus de 1000 espèces différentes (George 1993a, b). Les techniques de cultures de tissus sont d'un grand intérêt pour la collecte, la multiplication et la conservation du matériel génétique (Engelmann, 1991). Les systèmes de culture de tissus permettent de propager le matériel végétal avec des taux de multiplication élevés, dans un environnement aseptique. Des plantes exemptes de virus peuvent être obtenues par culture de méristèmes en combinaison avec la thermothérapie, ce qui permet la production de stocks exempts de virus et simplifie les procédures de quarantaine pour l'échange international de matériel génétique. La miniaturisation des explants permet de réduire l'espace nécessaire pour la conservation, et, par conséquent, de réduire les coûts de main-d'œuvre pour l'entretien des collections de matériel génétique. Différentes techniques de conservation in vitro sont utilisées selon la durée de stockage recherchée (Engelmann, 1991 ; Krishnapillay et Engelmann, 1996). Pour le stockage à court et moyen terme, on utilise les techniques de conservation en croissance ralentie. Pour la conservation à long terme, la cryoconservation, c'est-à-dire le stockage à température ultrabasse, généralement celle de l'azote liquide $\left(-196^{\circ} \mathrm{C}\right)$, est la seule méthode utilisable. Les techniques de collecte in vitro, de conservation en croissance ralentie et de cryoconservation sont décrites dans les sections suivantes.

\section{Collecte in vitro}

La collecte de matériel génétique d'espèces à semences récalcitrantes ou à multiplication végétative peut poser différents problèmes qui peuvent conduire à la perte du matériel collecté à cause de sa détérioration, de sa contamination ou de sa viabilité réduite. Ces problèmes peuvent être surmontés si l'on réalise que la semence n'est pas le seul matériel qui peut être collecté : les embryons zygotiques ou les tissus végétatifs comme des fragments de tige, des bourgeons ou des apex peuvent être prélevés, transportés et cultivés avec succès s'ils sont placés dans les conditions adéquates. À la suite d'une réunion d'experts organisée par l'IBPGR ${ }^{1}$ en 1984 et le financement de divers programmes de recherche, des techniques de collecte in vitro ont été développées pour différents matériels tels que les embryons de cocotier, de cacaoyer, d'avocatier, d'agrumes, des organes végétatifs de cacaoyer, de Musa spp., de caféier, de Prunus, de vigne, de cotonnier et de diverses espèces fourragères (Pence et al., 2002).

\section{Conservation en croissance ralentie}

Pour la croissance en vie ralentie, la technique la plus largement utilisée est la réduction de la température qui peut être combinée avec une diminution de l'intensité lumineuse ou avec une culture à l'obscurité. Des températures de l'ordre de $0-5{ }^{\circ} \mathrm{C}$ sont employées pour les espèces tolérantes au froid. Les espèces tropicales sont souvent sensibles au froid et doivent être conservées à des températures plus élevées, qui dépendent de leur sensibilité au froid. Diverses modifications peuvent également être apportées au milieu de culture afin de ralentir la croissance (Withers et Engelmann, 1998). Les techniques de stockage in vitro en croissance ralentie sont utilisées en routine pour la conservation à moyen terme de nombreuses espèces, à la fois d'origine tropicale et tempérée, telles que la pomme de terre, les Musa spp., l'igname et le manioc (Engelmann, 1999). En 1996, la FAO recensait environ 38000 accessions conservées in vitro en vie ralentie (FAO, 1996). Cependant, si la conservation in vitro semble une option simple et pratique pour la conservation à moyen terme de nombreuses espèces, son utilisation nécessite une adaptation à chaque nouveau matériel ainsi que des apports continus d'intrants, et des questions se posent quant à la stabilité génétique du matériel stocké pour certaines espèces. Des directives techniques ont été publiées récemment (Reed et al., 2004) qui peuvent servir de guide aux chercheurs et gestionnaires des banques de gènes pour l'établissement et la gestion des collections in vitro de ressources génétiques.

\section{Cryoconservation}

À la température de l'azote liquide, toutes les divisions cellulaires sont stoppées et le métabolisme arrêté. Le matériel végétal peut ainsi être conservé sans altération ni modification pendant des durées théo-

\footnotetext{
${ }^{1}$ International Board for Plant Genetic Resources (maintenant Bioversity International, IPGRI)
}

riquement illimitées. De plus, les cultures sont stockées dans un volume réduit, à l'abri des contaminations et avec un entretien limité. Il est important de réaliser que la cryoconservation est la seule technique disponible à l'heure actuelle permettant la conservation économique, en sécurité, des ressources génétiques du matériel végétal dont la conservation pose des problèmes.

Certains matériels comme les semences orthodoxes ou les bourgeons dormants présentent des processus naturels de déshydratation et peuvent être cryoconservés sans aucun prétraitement. Cependant, la plupart des systèmes expérimentaux employés en cryoconservation (bourgeons, embryons, suspensions ou cals) contiennent des quantités d'eau intracellulaire élevées et sont donc extrêmement sensibles à la congélation. Les cellules doivent donc être déshydratées artificiellement pour les protéger des dégâts causés par la cristallisation de l'eau intracellulaire pour former de la glace. Les techniques employées et les mécanismes physiques sur lesquels elles reposent sont différents dans les techniques de cryoconservation classiques et nouvelles (Withers et Engelmann, 1998). Les techniques classiques sont fondées sur la déshydratation pendant la congélation, alors que les nouvelles techniques le sont sur la vitrification. La vitrification peut être définie comme la transition de l'eau directement de la phase liquide en une phase amorphe ou verre, en évitant la formation de glace cristalline dommageable pour l'intégrité cellulaire. Les techniques de cryoconservation classiques ont été appliquées avec succès aux systèmes indifférenciés tels que les suspensions cellulaires et les cals (Withers et Engelmann, 1998). Dans le cas des cultures différenciées, ces techniques peuvent être employées seulement pour la congélation d'apex d'espèces tolérantes au froid. Dans les procédures fondées sur la vitrification, la déshydratation cellulaire est réalisée avant la congélation en exposant les échantillons à des solutions cryoprotectrices extrêmement concentrées ou à la dessiccation physique. La déshydratation est suivie par la congélation. Avec ces techniques, tous les problèmes liés à la formation de glace intracellulaire sont évités. Les procédures fondées sur la vitrification offrent des avantages pratiques par rapport aux techniques classiques. Elles sont plus appropriées aux organes complexes tels que les bourgeons ou les embryons qui ont une structure histologique hétérogène. 
En empêchant la formation de glace dans le système, les protocoles fondés sur la vitrification sont de mise en œuvre moins complexe que les protocoles classiques (ils ne nécessitent pas de congélateurs programmables) et ils ont un potentiel d'applicabilité beaucoup plus large (Engelmann, 1997). Sept techniques différentes fondées sur la vitrification ont été mises au point : encapsulation-déshydratation, vitrification, encapsulation-vitrification, déshydratation, préculture, préculture-déshydratation et vitrification en gouttes (Engelmann, 2004; Gonzalez-Arnao et Engelmann, 2006; Sakai et Engelmann, 2007). Ces nouvelles techniques ont été utilisées pour la cryoconservation de bourgeons et d'embryons de nombreuses espèces végétales d'origine tropicale et tempérée (Engelmann et Takagi, 2000 ; Reed, 2008).

Bien que leur utilisation en routine soit encore limitée, il existe un nombre croissant d'exemples pour lesquels la cryoconservation est employée à grande échelle avec différents types de matériels, tolérants ou non à la dessiccation. Ces matériels comprennent des semences d'espèces orthodoxes ou récalcitrantes, des bourgeons dormants, du pollen, des produits des biotechnologies et des bourgeons prélevés sur des vitroplants (Engelmann, 2008). Ces progrès ont été rendus possibles par le développement des nouvelles techniques fondées sur la vitrification qui ont permis son application à une large gamme d'espèces (Engelmann, 2004). Un avantage important de ces nouvelles techniques est leur simplicité de mise en œuvre, puisqu'elles sont destinées à être utilisées dans les pays tropicaux, dans lesquels la plus grande partie des ressources génétiques des espèces posant des problèmes de conservation est située. Pour un nombre non négligeable d'espèces à multiplication végétative, les techniques de cryoconservation sont suffisamment avancées pour pouvoir envisager leur utilisation immédiate en routine dans les banques de gènes. Les recherches sont nettement moins avancées pour les espèces à semences récalcitrantes. Cela est dû au nombre très important de ces espèces, qui sont principalement sauvages, et au nombre limité d'activités de recherche visant à améliorer leur conservation. Cependant, il existe différentes approches techniques pour améliorer leur efficacité et augmenter leur applicabilité aux espèces récalcitrantes. De plus, des recherches sont activement conduites par différents groupes dans le monde pour augmenter les connaissances sur les mécanismes biologiques et physiques sur lesquels est fondée la récalcitrance. Des résultats nouveaux sur des points clés comme la compréhension et le contrôle de la sensibilité à la dessiccation contribueront de manière significative au développement de techniques de cryoconservation améliorées pour les espèces à semences récalcitrantes. À ce sujet, il est intéressant de mentionner qu'un projet COST (European Cooperation in the field of Scientific and Technical Research), financé par l'Union européenne - action COST 871 : "Cryopreservation of crop species in Europe "-, a été initié récemment. Cette action vise notamment à accroitre les connaissances fondamentales sur la cryoprotection par la détermination des changements physico-biochimiques associés avec la tolérance à la cryoconservation et à développer et à appliquer de nouveaux protocoles de cryoconservation ${ }^{2}$. On peut donc s'attendre à ce que, dans les années qui viennent, notre compréhension des mécanismes impliqués dans la cryoconservation augmente et que la cryoconservation devienne plus fréquemment employée pour la conservation à long terme des ressources phytogénétiques.

\section{Conclusion}

Dans cet article, nous avons présenté les nouvelles possibilités offertes par les biotechnologies pour améliorer la conservation ex situ de la diversité végétale. Des progrès très importants ont été faits au cours des dernières années, avec le développement de nouvelles techniques de conservation pour les espèces à semences non orthodoxes et à multiplication végétative, particulièrement dans le domaine de la cryoconservation. Les concepts actuels de la conservation ex situ devront être modifiés en conséquence pour intégrer ces avancées technologiques.

Il est maintenu reconnu qu'une stratégie de conservation appropriée à un pool génétique particulier nécessite une approche holistique, qui combine de manière complémentaire les différentes techniques de conservation in situ et ex situ disponibles. Les méthodes in situ et

\footnotetext{
2 Pour plus d'informations, le site web http:// www.biw.kuleuven.be/dtp/tro/cost871/ Home.html peut être consulté.
}

ex situ, qui incluent une gamme de techniques pour cette dernière, sont des options disponibles pour les différents composants de ce pool génétique. La sélection des méthodes appropriées doit être fondée sur un ensemble de critères, incluant la nature biologique de l'espèce considérée, la faisabilité des méthodes particulières choisies (qui dépendent de la disponibilité des infrastructures adéquates), de même que la sécurité et la rentabilité économique apportées par leur application. Les aspects de complémentarité en ce qui concerne l'efficacité et le coût des différentes méthodes de conservation choisies sont aussi importants. Dans de nombreux cas, le développement des stratégies complémentaires de conservation appropriées nécessitera encore des recherches supplémentaires pour définir les critères, optimiser les méthodes et tester leur application pour une gamme de pools génétiques et situations. Dans ce contexte, il est important de souligner que ces nouvelles techniques de conservation in vitro très efficaces ne sont pas perçues comme destinées à remplacer les approches conventionnelles de conservation ex situ. Elles offrent aux gestionnaires des banques de gènes et aux curateurs de jardins botaniques des outils supplémentaires pour leur permettre d'améliorer la conservation du matériel génétique placé sous leur responsabilité.

\section{Références}

Engelmann F. In vitro conservation of tropical plant germplasm - a review. Euphytica 1991 . $57: 227-43$.

Engelmann F. In vitro conservation methods. In: Ford-Lloyd BV, Newburry JH, Callow JA, eds. Biotechnology and Plant Genetic Resources: Conservation and Use. Wellingford : CABI, 1997.

Engelmann F. Management of field and in vitro germplasm collections. Proceedings of a Consultation Meeting, January, 1996, CIAT, Cali, Colombia. Rome: International Plant Genetic Resources Institute, 1999.

Engelmann F. Plant cryopreservation: progress and prospects. In vitro Cell Dev Biol - Plant $2004 ; 40: 427-33$.

Engelmann F. Use of biotechnologies for conserving plant biodiversity. Acta Hortic 2008 sous presse.

Engelmann F, Takagi H. Cryopreservation of Tropical Plant Germplasm - Current Research Progress and Applications. Tsukuba : JIRCAS Rome: IPGRI, 2000. 
FAO. Report on the State of the World's Plant Genetic Resources for Food and Agriculture. Rome: Food and Agriculture Organization of the United Nations, 1996.

George EF. Plant Propagation by Tissue Culture. Part 1 The Technology. Second Edition. Edington : Exegetics Ltd., 1993a.

George EF. Plant Propagation by Tissue Culture. Part 2 In Practice. Second Edition. Edington : Exegetics Ltd., 1993b.

Gonzalez-Arnao MT, Engelmann F. Cryopreservation of plant germplasm using the encapsulation-dehydration technique: review and case study on sugarcane. CryoLetters $2006 ; 27: 155-68$
Krishnapillay DB, Engelmann F. Alternative methods for the storage of recalcitrant and intermediate seeds: slow growth and cryopreservation. In: Oueddraogo AS, Poulsen K, Stubsgaard F, eds. Proceedings of the Workshop on Improved Methods for Handling and Storage of Intermediate and Recalcitrant Forest Tree Seeds. Rome : IPGRI ; Humlebaek, (Danemark): Danida Forest Seed Centre, 1996.

Pence VC, Sandoval J, Villalobos V, Engelmann F. In vitro Collecting Techniques for Germplasm Conservation. IPGRI Technical Bulletin N ${ }^{\circ}$ 7. Rome: IPGRI, 2002.

Reed BM. Plant Cryopreservation - A Practical Guide. New York: Springer, 2008.
Reed $\mathrm{BM}$, Engelmann $\mathrm{F}$, Dulloo ME, Engels JMM. Technical Guidelines for the Management of Field and In vitro Germplasm Collections. Handbook for Genebanks N ${ }^{\circ}$. Rome: IPGRI/SGRP, 2004.

Roberts HF. Predicting the viability of seeds. Seed Sci Technol 1973; 1: 499-514.

Sakai A, Engelmann F. Vitrification, encapsulation-vitrification and dropletvitrification: a review. CryoLetters 2007; 28 : 151-72.

Withers LA, Engelmann F. In vitro conservation of plant genetic resources. In : Altman A, ed. Biotechnology in Agriculture. New York: Marcel Dekker Inc, 1998. 